\title{
On the geometry of sunspot penumbral filaments
}

\author{
W. Schmidt and G. Fritz \\ Kiepenheuer-Institut für Sonnenphysik, Schöneckstraße 6, 79104 Freiburg, Germany \\ Received 2 February 2004 / Accepted 15 March 2004

\begin{abstract}
We investigate the center-to-limb variation of the brightness of the penumbrae of sunspots. The analysis includes narrow-band and broad-band continuum images of about 80 sunspots observed with the Vacuum Tower Telescope on Tenerife and with the Transition Region And Coronal Explorer (TRACE). We find that the azimuthal intensity variation depends on the viewing angle, i.e. on the position of the spot on the solar disk. With increasing viewing angle, the average intensity remains almost constant on the center side and on the limb side of the spot, but increases in the lateral parts. This is explained by the existence of elevated bright filaments that, when observed from the side and at increasing viewing angle, more and more hide the dark and deeper lying background. In addition to this azimuthal intensity modulation, we observe an enhanced brightness on the limb-side penumbra for large viewing angles.
\end{abstract}

Key words. Sun: sunspots

\section{Introduction}

The topography of the penumbra of sunspots is important for understanding the physical mechanisms that are responsible for the formation, the kinematics and the typical time scales of these well-known, but still enigmatic objects on the solar surface. A host of observations exists, with quite inconclusive results, see e.g. Westendorp Plaza et al. (2001) or the reviews of Solanki (2003) or Bellot Rubio (2004). While Sütterlin (2001) claims to have spatially resolved the penumbral fibrils, recent observations with the new Swedish solar telescope (Scharmer et al. 2002) have revealed new details of the spot morphology at spatial scales below $100 \mathrm{~km}$ on the sun, and evidence for still unresolved features.

Observations of the magnetic configuration and the kinematic properties have indicated that the dark filaments may be elevated relative to the bright ones (Rimmele 1995; or Stanchfield et al. 1997). The results of Hirzberger \& Kneer (2001) and Schlichenmaier et al. (2004) do not support that picture. Rouppe van der Voort (2002) shows that penumbral fibrils that are bright in the lower photosphere remain so over a height range of a few hundred $\mathrm{km}$, i.e. there is no contrast reversal with height. The idea of elevated penumbral filaments has already been mentioned by Moore (1981), based on the investigation of sunspot photographs. Solanki \& Montavon (1994) used a similar picture as a possible explanation for the brightness variation at the umbra-penumbra border, but, to our knowledge, no observations have been carried out in this respect.

Send offprint requests to: $\mathrm{W}$. Schmidt, e-mail: wolfgang@kis.uni-freiburg.de
The present work concentrates on the topography of penumbral filaments observed in the continuum. We address the question of whether or not bright filaments are elevated relative to the dark ones. We concentrate on continuum measurements and investigate geometrical projection effects. This should allow us to distinguish between apparent height variations caused by opacity effects and between geometrical height differences. To this end, we measure the azimuthal intensity modulation of sunspot penumbrae and study the center-to-limb variation of this modulation. In order to overcome the inherent variability of the highly structured penumbrae and to obtain a statistically significant data set, we investigate more than 80 sunspots. In Sect. 2 we describe the data sets and the method of analysis. The results are presented and discussed in Sects. 3 and 4.

\section{Data analysis}

The analysis is based on several independent data sets. Firstly we used 55 white light images observed with the Transition Region And Coronal Explorer (TRACE) satellite between 1998 and 2001. The second data set consists of 33 sunspot maps, taken with the Tenerife Infrared Polarimeter (TIP) at the Vacuum Tower Telescope (VTT) in the Observatorio del Teide on Tenerife. The main characteristics of the data sets are summarized in Table 1. More information about TRACE and TIP can be found in Strong et al. (1994) and Martínez Pillet et al. (1999). The TRACE data are broadband images, while the TIP data are narrowband rasters taken in the spectral continuum near the Fe I $1565 \mathrm{~nm}$ line. Figure 1 shows examples for different heliocentric angles, taken from the TRACE data set. 
Table 1. Main characteristics of the data sets: instrument, No. of images, wavelength and pixel size.

\begin{tabular}{lccc}
\hline \hline Instrument & No. of spots & $\lambda[\mathrm{nm}]$ & Pixel [arcsec] \\
\hline TRACE & 55 & 500 & 0.5 \\
TIP-1 (VTT) & 13 & 1565 & 0.4 \\
TIP-2 (VTT, 2002) & 20 & 1565 & 0.4 \\
\hline
\end{tabular}

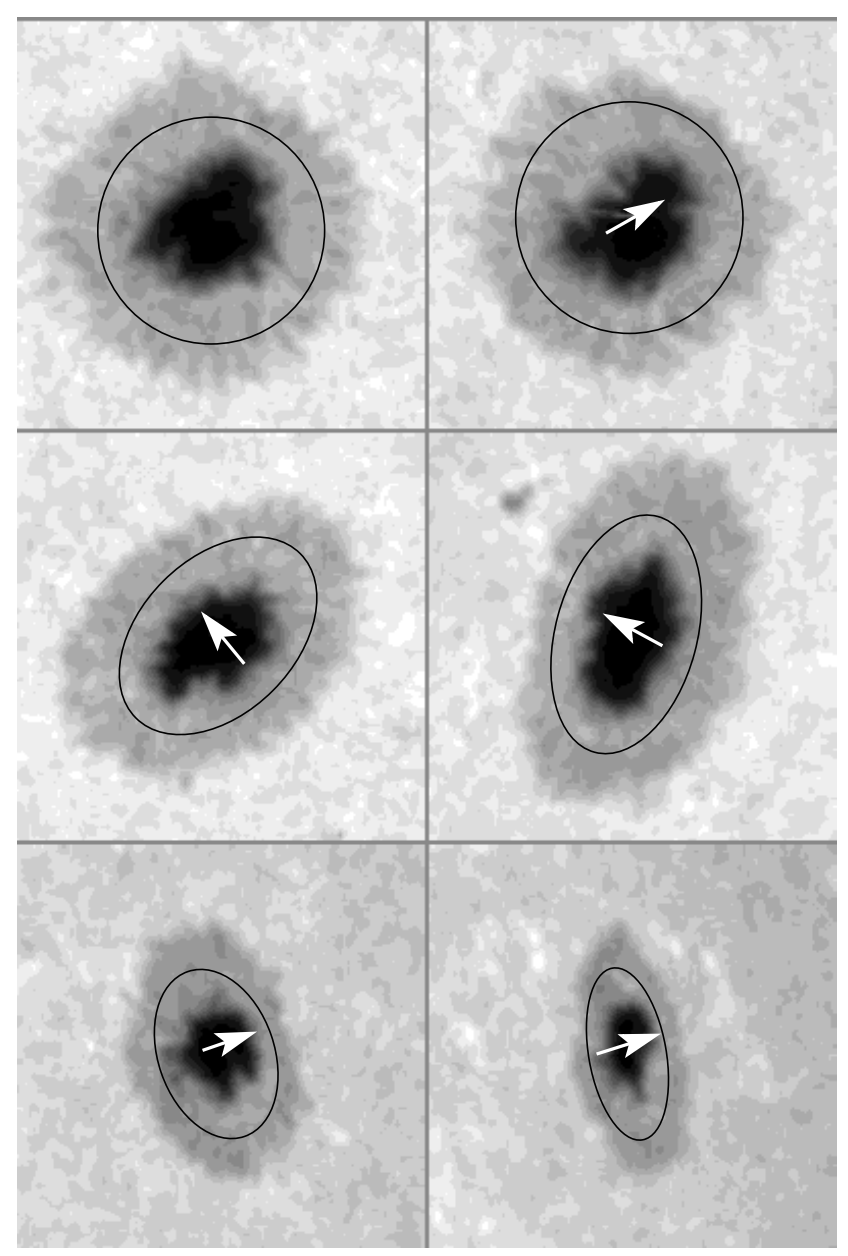

Fig. 1. Examples of spots observed at different disk positions. Top to bottom, left to right: $\mu=1,0.9,0.8,0.7,0.6,0.5$. The paths used for the intensity tracings are indicated. The arrows point towards disk center.

\subsection{Image selection}

We selected roundish and regular-shaped sunspots with well developed penumbrae, located at different positions on the solar disk, i.e. seen at different viewing angles. The target selection for the TRACE data was made on the basis of synoptic images taken with the Solar and Heliospheric Observatory (SOHO). The TRACE images have a pixel size of $0.5^{\prime \prime}$ and are of constant quality. The continuum maps of TIP were byproducts of observing campaigns carried out between 1999 and 2003 and independent of the present investigation. The sunspot images for this analysis have been selected by visual inspection. For a proper comparison of the azimuthal intensity variation of the penumbral brightness, it is necessary to

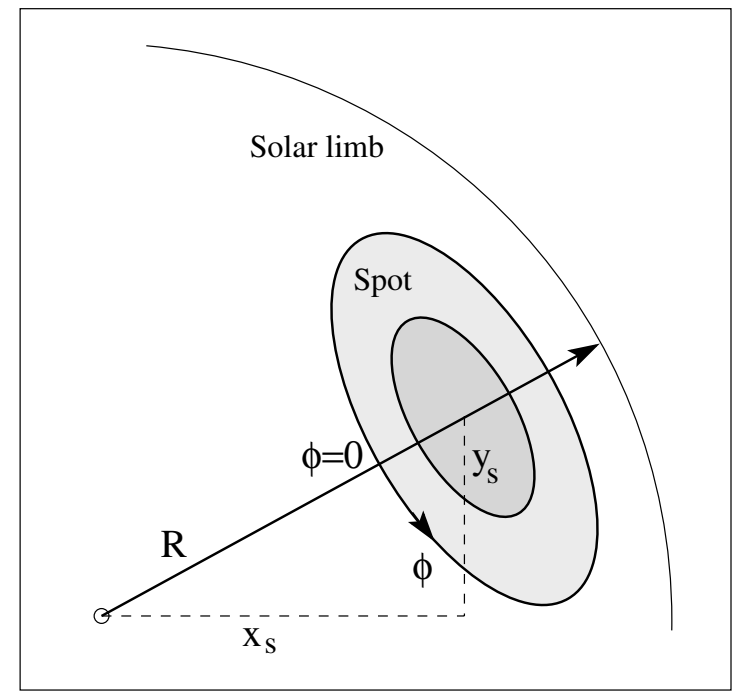

Fig. 2. Coordinate system used for the data analysis. $R$ is the apparent radius of the solar disk, $x_{\mathrm{s}}$ and $y_{\mathrm{s}}$ denote the disk coordinates of the spot center. $\phi$ is the spot azimuth, measured counter-clockwise from the center-side intersection of the spot circumference with the line connecting the center of the spot and the center of the solar disk.

establish the orientation of each spot on the solar disk. The azimuth angle $\phi$ is counted counter-clockwise around the spot, starting from the center side, i.e. the intersection of the spot circumference with the line connecting the spot center and the center of the solar disk. The TRACE image data contain the disk coordinates $\left(x_{0}, y_{0}\right)$ of the center of each image. The location of the spot center within each picture is given by the coordinates $x_{\mathrm{c}}, y_{\mathrm{c}}$, and the disk coordinates of the center of the spot are $x_{\mathrm{s}}=x_{0}+x_{\mathrm{c}}$, and $y_{\mathrm{s}}=y_{0}+y_{\mathrm{c}}$ (see Fig. 2). The heliocentric angle, $\theta$, is then derived from these coordinates (expressed in $\operatorname{arcsec})$ as

$\theta=\arcsin \frac{\sqrt{\left(x_{0}+x_{\mathrm{c}}\right)^{2}+\left(y_{0}+y_{\mathrm{c}}\right)^{2}}}{R_{\odot}}$.

In the following we use the parameter $\mu=\cos \theta$ to characterize the disk position of the analyzed sunspots.

\subsection{Analysis procedure}

Our analysis is rather simple and straightforward, and it is especially insensitive to the spatial resolution. The underlying picture is as follows: the penumbral filaments are elongated in radial direction and bright and dark filaments have similar width, but the respective filling factor does not need to be equal. If the bright filaments are elevated with respect to the dark ones by a certain amount, they would partially hide the deep-lying dark ones, if seen from the side. The degree of occultation would depend upon the viewing angle, and beyond a critical angle, the elevated component would completely hide the dark background. In that case, the measured brightness would be that of the bright filaments. This effect, if it existed, would be a function of the azimuth angle, measured from spot center. In those parts of the penumbra, where the view is more or less parallel to the line-of-sight, one would always see the bright and dark 


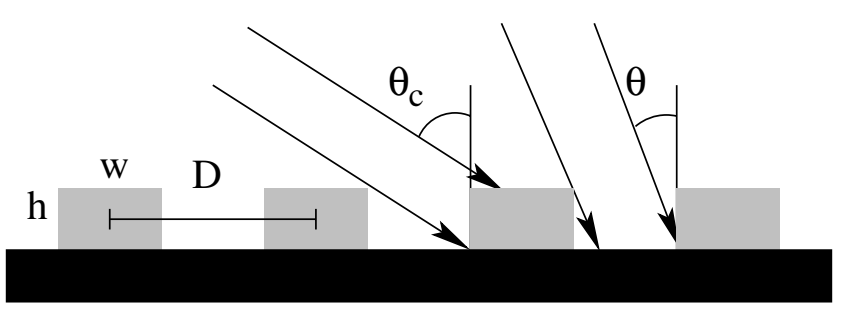

Fig. 3. Scheme of elevated bright filaments of width $w$, separation $D$ and height $h$. The angle $\theta$ is the heliocentric angle of the spot.

filaments, resolution permitting, or measure the averaged intensity of the bright and dark component. In the lateral parts of the spot, i.e. around $\phi \approx 90^{\circ}$ and $\phi \approx 270^{\circ}$, the shading or occultation effect would occur. A schematic description of this effect is shown in Fig. 3. The picture addresses the simplest case with the line-of-sight perpendicular to the filaments.

We use the simple geometry shown in Fig. 3 to estimate the observed brightness of the penumbra, $I_{0}$, as a function of spot azimuth, $\phi$, and the heliocentric angle, $\theta$. The measured intensity. $I_{0}$ depends on the brightness of the bright and dark components, $I_{\mathrm{b}}$ and $I_{\mathrm{d}}$, the elevation $h$ of the bright filaments relative to the dark background, the angle $\theta$, the width $w$ of the bright filaments and the distance $D$ between adjacent bright filaments as follows:

$I_{\mathrm{o}}=\frac{1}{D}\left(I_{\mathrm{b}}(w+h \tan \theta \sin 2 \phi)+I_{\mathrm{d}}(D-w-h \tan \theta \sin 2 \phi)\right)$.

This simple expression is only valid until the quantity $h \tan \theta$ equals the width of a dark filament, $D-w$, i.e. until the dark background is completely hidden by the bright filaments and the observed mean intensity equals that of the bright filaments. Figure 4 shows $I_{\mathrm{O}}$ as a function of the sunspot azimuth angle for different values of the heliocentric angle. The height, $h$, of the filaments was chosen to be $10 \%$ of the width, $w$.

\subsection{Intensity curves}

The intensity as a function of spot azimuth was extracted from each sunspot along circles or ellipses placed at a constant distance from the umbra-penumbra boundary. The position, orientation and shape of the ellipses has been adapted by hand for each spot under investigation. We used the average of at least two confocal ellipses with slightly different major axes, placed in the middle penumbra for the analysis. The ellipses have been adapted to the geometry of each spot under investigation, independent of its heliographic angle. This allowed to include also non-spherical spots. The resulting intensity curves then have been arranged in six groups, according to their heliocentric angles, from disk center to the limb. The results for the three different sets of spots are shown separately in Fig. 5. The $\mu$-intervals are given in the panel-headers of Fig. 5 .

\section{Results}

\subsection{Intensity modulation}

The main result of the analysis is a modulation of the penumbral intensity measured along an azimuthal path in the middle

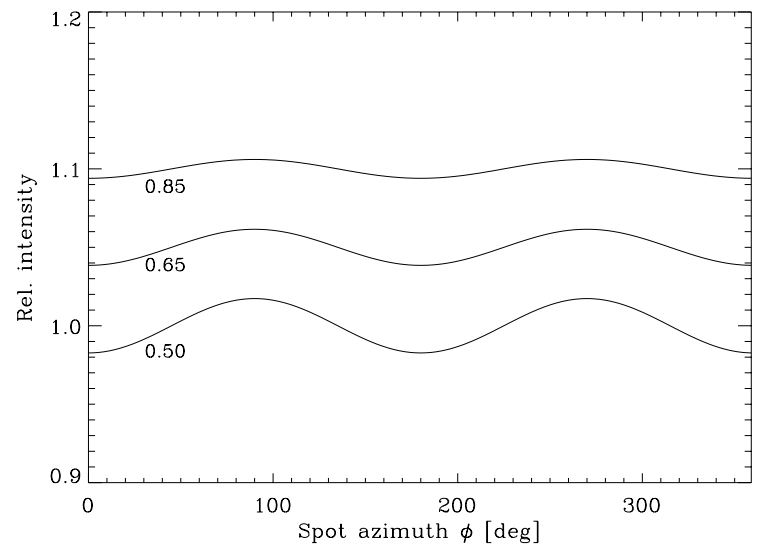

Fig. 4. Modulation of the mean penumbral intensity, measured as a function of spot azimuth angle, using Eq. (2), for different heliocentric angles. The height of the bright filaments is $10 \%$ of the filament width in this example. The labels give $\mu=\cos \theta$.

penumbra. The amplitude of this modulation depends on the heliocentric position of the spot, i.e. of the viewing angle between the observed spot and the local surface normal. The modulation also depends on the precise location of the track. In the present work we concentrate on the middle penumbra, because that part of a sunspot penumbra is most regular and disturbances from the outer or inner penumbral boundary (e.g. light bridges) play a minor role. Figure 5 summarizes the results. It shows the penumbral intensity as a function of spot azimuth angle. The six panels of the Figure correspond to intervals of heliocentric angles, starting from disk center, $1.0>\mu>0.95$, until the vicinity of the solar limb, $0.5>\mu>0.2$. Each panel shows the averages of all spots that belong to the same group, but separately for the three data sets (TRACE, TIP-1, TIP-2). The intensity curves have been shifted along the vertical axis by a fixed amount for better legibility. The intensity curves have been normalized to one prior to any averaging. Near disk center, and at heliocentric angles below 30 degrees (first two panels), there is no systematic or consistent effect: some data show a large-scale modulation, others do not. Between 40 and 50 degrees, a consistent modulation pattern builds up: the intensity is increased around azimuth angles 90 and 270 degrees, but remains unchanged near 0 and 180 degrees. The intensity modulation, $I_{\mathrm{m}}=I-I_{\text {mean }}$, is more or less sinusoidal, with $I_{\mathrm{m}} \sim \sin 2 \phi$. The amplitude of the modulation grows with increasing heliocentric angle (panels 5 and 6), and the phase of the modulation remains constant. The intensity is highest at azimuth angles of $90^{\circ}$ and $270^{\circ}$, where the filaments are observed from the side. The center-side $\left(\phi=0^{\circ}\right)$ and limb-side penumbra $\left(\phi=180^{\circ}\right)$ virtually maintain their brightness, independently of disk position, because they are observed parallel to their orientation, and only perspective foreshortening occurs. The dashed curves show the second (complex) Fourier component derived from the respective measurement (TRACE, TIP-1, TIP-2). The corresponding phase angles confirm the visual impression. The modulation effect is small, but it is seen consistently in the three - totally independent and different - data sets. 

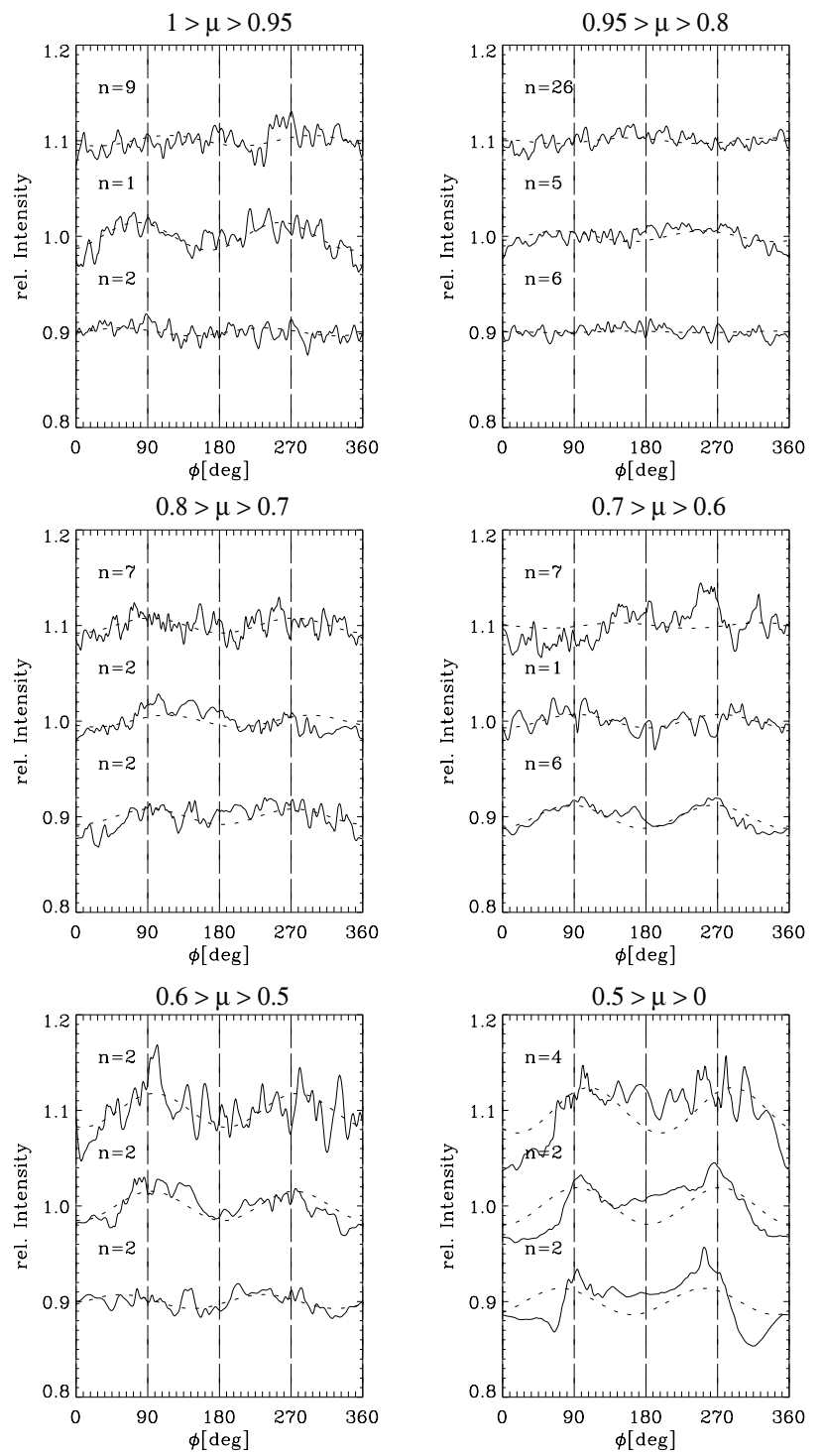

Fig. 5. Azimuthal variation of penumbral brightness, measured along ellipses with constant distance to the inner penumbral boundary. The six panels correspond to different intervals $\mu=\cos \theta$ of the sunspots. The three curves in each panels show the average behavior for all sunspots within the $\mu$-interval, separately for the three data sets under investigation. The number, $n$, of sunspots in each interval is indicated. Top curve: TRACE, middle curve: TIP-1, bottom curve: TIP-2. $\phi=0$ is at center-side (cf. Fig. 2). The dashed line is the 2nd Fourier component of the corresponding curve. The three sets of curves are shifted in intensity by 0.1 units, for clarity.

\subsection{Error estimate}

The largest error source is certainly the intrinsic intensity variation of the penumbra itself. In addition to the filamentary pattern, the intensity may also change with radius. A nice, regular and axially symmetric intensity pattern is seldom found. In order to minimize this effect, we selected by eye the most regular spots, with fully developed penumbrae and no light bridges. The second uncertainty arises from the proper placement of the ellipses within the penumbrae. Since the azimuthally averaged penumbral brightness varies with radius, any misplacement of the elliptical path would cause an apparent azimuthal intensity modulation. That effect would, however, produce a modulation varying with $\sin \phi$, while the obscuration effect varies like $\sin 2 \phi$. In addition, both error sources described above are statistical ones in the sense that no preferred phase angles for the azimuthal variation exists. It is therefore highly unlikely that the observed intensity modulation is produced by the method of analysis itself.

\section{Discussion}

A straightforward explanation of our results is a certain elevation of the bright penumbral filaments compared to the dark background. On the center and on the limb side, the (projected) line-of sight is more or less parallel to the filaments and eventual height differences cannot be seen. With increasing viewing angle, and when seen from the side, the elevated bright structures more and more hide the dark background. This shadowing effect leads to an increase of the bright area, and consequently to an increased mean intensity. This effect is virtually independent of the spatial resolution of the observations.

In the present paper we have shown the existence of elevated bright filaments on the basis of a geometrical effect. This implies that the elevated structures have indeed to be hotter and that the increased brightness cannot be caused by a decreased opacity that would allow to see deeper and hotter layers. The increased temperature of the bright filaments causes an increase of the continuum opacity, due to the increased electron density. Therefore, the continuum level is shifted upwards, in agreement with our findings. From a comparison of the intensity modulation computed from our simple filament model shown in Fig. 4 with our results (Fig. 5), we estimate an elevation of about $10 \%$ of the width of the filaments.

We note that a completely flat, two-dimensional penumbra is an unlikely configuration, and the observed threedimensional structure was to be expected, at least to some degree. Model calculations, e.g. by Schlichenmaier et al. (1998), or Montesinos \& Thomas (1997) predict the existence of elevated flow channels. The interpretation of dark penumbral fibrils being elevated with respect to the bright ones given by Moore (1981) is not compatible with our results. It might be worth while to reconsider the work of Cram et al. (1981), who asserted the existence of elevated dark filaments and had tried to model them.

It is difficult to compare our results, obtained in the spectral continuum (i.e. at optical depth unity), with spectroscopic measurements, e.g. those of Rimmele (1995). The existence of elevated flow channels does not necessarily imply that the cospatial dark filaments are also elevated relative to the bright ones.

The spots located closest to the limb (panel at lower right in Fig. 5) show an additional feature: in all data sets, the intensity at the limb side $\left(\phi=180^{\circ}\right)$ is higher than at the center side of the spot. This result may be due to an opacity effect: on the limb side, the continuum level, $\tau=1$, is reached in a slightly deeper layer, compared to the center side penumbra, because of the Wilson depression of the spot and the different inclination angles of the center and limb side of the 
penumbra. The difference in inclination is a few degrees (with a Wilson depression of $400 \mathrm{~km}$ and a $6000 \mathrm{~km}$ wide penumbra, we have $\delta=\arctan 1 / 15=3.8^{\circ}$ ). Note that this effect has the opposite sign to the general center-to-limb variation of the continuum. Our findings are in qualitative agreement with the results of Westendorp Plaza et al. (2001), who found from their velocity measurements that deeper (i.e. hotter) layers are probed on the limb-side penumbra. In contrast thereto, Rouppe van der Voort (2002) and Schlichenmaier et al. (2004) found a slightly higher temperature of the center-side penumbra compared to the limb side. Note that our findings are based on spots at heliocentric angles larger than 60 degrees, whereas the other authors cited in this paragraph have analyzed spots at viewing angles of about 30 degrees.

Our analysis and hence the results are independent of spatial resolution. It is nevertheless necessary and important to perform a similar investigation with high spatial resolution in order to clarify the influence of penumbral grains and other smallscale objects on the center-to-limb variation of the penumbral brightness.

Acknowledgements. Rolf Schlichenmaier and Luis Bellot Rubio put some of their data at our disposal. We thank Alexandra Tritschler, Luis Bellot Rubio and Rolf Schlichenmaier for useful comments and suggestions. P. Caligari helped to customize the software needed to use the TRACE data archive at the KIS.

\section{References}

Bellot Rubio, L. 2004, Rev. Mod. Astron., 17, 21

Cram, L. E., Nye, A. H., \& Thomas, J. H. 1981, in The Physics of Sunspots, 384

Hirzberger, J., \& Kneer, F. 2001, A\&A, 378, 1078

Martínez Pillet, V., Collados, M., Sánchez Almeida, J., et al. 1999, in High Resolution Solar Physics: Theory, Observations, and Techniques, ASP Conf. Ser., 183, 264

Montesinos, B., \& Thomas, J. H. 1997, Nature, 390, 485

Moore, R. L. 1981, ApJ, 249, 390

Rimmele, T. R. 1995, A\&A, 298, 260

Rouppe van der Voort, L. H. M. 2002, A\&A, 389, 1020

Scharmer, G. B., Gudiksen, B. V., Kiselman, D., Löfdahl, M. G., \& Rouppe van der Voort, L. H. M. 2002, Nature, 420, 151

Schlichenmaier, R., Bellot Rubio, L. R., \& Tritschler, A. 2004, A\&A, 415,731

Schlichenmaier, R., Jahn, K., \& Schmidt, H. U. 1998, ApJ, 493, L121

Solanki, S., \& Montavon, C. 1994, in Solar Surface Magnetism, ed. R. Rutten, \& C. Schrijver, 239

Solanki, S. K. 2003, A\&AR, 11, 153

Stanchfield, D. C. H., Thomas, J. H., \& Lites, B. W. 1997, ApJ, 477, 485

Strong, K., Bruner, M., Tarbell, T., Title, A., \& Wolfson, C. J. 1994, Space Sci. Rev., 70, 119

Sütterlin, P. 2001, A\&A, 374, L21

Westendorp Plaza, C., del Toro Iniesta, J. C., Ruiz Cobo, B., et al. 2001, ApJ, 547, 1130 